\title{
New investigations of polyatomic molecules in the liquid state using picosecond light pulses
}

\author{
W. Zinth and W. Kaiser \\ Physics Department, Technical University, Munich, West Germany \\ (Submitted June 25, 1982) \\ Kvantovaya Elektron. (Moscow) 10, 44-52 (January 1983) \\ A description is given of a new method for investigating vibrational excitations of molecules in liquids: this \\ method involves a preliminary brief excitation of a molecule by two picosecond pulses, differing in frequency \\ by an amount which is in resonance with natural vibrations of the molecule, followed by a subsequent \\ prolonged investigation of free relaxation of the excited system. The results are reported of an investigation of \\ this kind made on $\mathrm{C}_{6} \mathrm{H}_{12}$ in the range $2875-2925 \mathrm{~cm}^{-1}$. Lines not resolved in the usual spontaneous Raman \\ spectrum were observed. The position of the well-known $2923 \mathrm{~cm}^{-1}$ line was determined more accurately.
}

PACS numbers: 78.30.Cp, 79.20.Ds

For many years the band contours of Raman transitions have been the main source of dynamic information on polyatomic molecules. However, in most cases it is difficult to separate the Raman band of interest from the background made up of other neighboring or weak overlapping Raman transitions. In the case of polyatomic molecules there are many overtones and combination modes which may contribute-possibly enhanced by a Fermi resonance-to the observed scatteredlight intensity.

When a band contour is determined accurately, difficulties are encountered in the interpretation of the physical origin of the Raman band width. In general, there are several line-broadening mechanisms which may or may not contribute to the observed Raman band. These include collision-induced dephasing, energy relaxation of excited vibrational states, possible isotopic multiplicity of a transition, and finally inhomogeneous broadening due to changes in the molecular environment.

Recently we were able to determine directly the population lifetime $T_{1}$ and the dephasing time $T_{2}$ of various vibrational modes in the time domain without recourse to the width of the vibrational transition lines. ${ }^{1-8}$ In the case of polyatomic molecules in the liquid state at $300^{\circ} \mathrm{K}$ both time constants $T_{1}$ and $T_{2}$ are very short, frequently of the order of picoseconds. Consequently, we had to design experimental systems to enable us to measure vibrational time constants on the time scale of $10^{-12} \mathrm{sec}$. In the present paper we shall review experimental techniques and report new results to illustrate the state of our knowledge of the population lifetime $T_{1}$. In addition we shall describe a novel coherent Raman technique which can be used to detect new Raman lines in congested spectral regions.

\section{POPULATION LIFETIME $T_{1}$}

The population lifetime of a specific vibrational state is measured as follows. The first ultrashort infrared pulse, turned to the resonance frequency of the investigated transition, excites molecules by direct infrared absorption, whereas the second visible pulse follows after a variable delay time and it monitors the instantaneous population of the excited molecules. A spon- taneous anti-Stokes Raman signal is used as a direct measure of the excess population. This technique has the great advantage of providing very direct information on the populated vibrational mode. Observation of various anti-Stokes frequencies tells us accurately which vibrational mode is populated at a certain time. In this way we are able to observe the time dependence of the primary excitation and to follow the vibrational energy through different decay relaxation) channels.

However, this technique for the determination of $T_{1}$ has a major shortcoming. The Raman scattering cross sections are notoriously very small and the population densities achieved after a short excitation pulse do not exceed 1\%. Consequently, the number of scattered photons is very small, approximately from $10^{2}$ to $10^{3}$ per pulse, which makes the data acquisition a nontrivial task. 4

\section{EXPERIMENTAL SYSTEM FOR MEASURING $T_{1}$}

Figure 1 shows schematically a more advanced experimental system for the determination of $T_{1}$. Two pulses differing considerably in frequency were generated. A tunable infrared pulse of frequency $\nu_{1}$ is generated by a three-photon process in three lithium niobate crystals. ${ }^{\circ}$ Typical intensities of infrared pulses were $10^{15}$ photons per pulse and the spectral band width was $\Delta \tilde{\nu}_{\mathrm{IR}} \approx 20 \mathrm{~cm}^{-1}$, and the beam divergence was

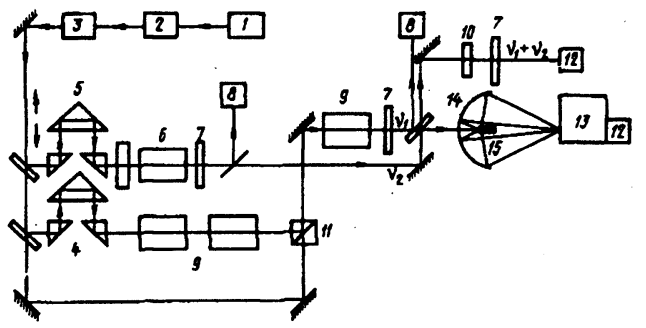

FIG. 1. Schematic diagram of the system used to measure the population lifetime $T_{1}$ of vibrational modes: 1) $Q$-switched laser; 2) switch; 3) amplifier; 4), 5) fixed and controlled optical delay línes; 6) KDP crystals; 7) optical filters; 8) photodiodes; 9) lithium niobate crystal; 10) lithium niobate plate; 11) polarizer; 12) photomultiplier; 13) spectrograph; 14) ellipsoldal mirror; 15) Investigated sample. 
$\sim 1 \mu \mathrm{rad}$. A monitoring green pulse $\left(\tilde{\nu}_{2}=18990 \mathrm{~cm}^{-1}\right)$ was generated in the form of the second harmonic frequency in a nonlinear KDP crystal. Both pulses were focused (after the selected delay time) in a small sample located at the focus of an ellipsoidal mirror. Approximately $50 \%$ of the anti-Stokes scattered photons were collected by this mirror and imaged at the entrance slit of a spectrometer connected to a photomultiplier. After different delays, both pulses were mixed in a thin lithium niobate crystal (10 in Fig. 1), generating the sum frequency. In this way we obtained the cross-correlation function for each experimental run. The peak of the cross-correlation function corresponded to zero point on our time scale (where the maxima of both pulses overlapped) and the width of the cross-correlation function yielded valuable information on the time resolution in each experiment.

\section{RECENT RESULTS AND DISCUSSION OF $T$, DATA}

We investigated numerous molecules and found widely varying values of the population lifetimes between 1 psec (corresponding to the time resolution of our system) and $200 \mathrm{psec}$ in the case of polyatomic molecules at room temperature.$^{1,2,4}$ Special attention was paid to the stretching modes of the $\mathrm{CH}$ groups in the frequency range $3000 \pm 100 \mathrm{~cm}^{-1}$, where direct pumping by infrared pulses was particularly effective.

At the beginning of our investigations we found the large spread in the $T_{1}$ values quite puzzling. The lifetimes $T_{1}$ appeared to be very sensitive to the specific level structure of the individual molecule. In the past few years we made a considerable progress in our understanding of the physical phenomena determining the population lifetime of polyatomic molecules. One should distinguish two processes.

The first process represents a very rapid exchange of the vibrational energy between similar fundamental vibrations with similar energies. For example, we have experimental evidence that collision-induced interactions lead to a rapid energy exchange (faster than $1 \mathrm{psec}$ ) between the symmetric and asymmetric stretching modes of the $\mathrm{CH}_{2}$ group. Consequently, excitation with pulses of 5 psec duration populates both vibrational levels. Indeed, a very fast energy exchange (faster than 1 psec) occurs between the infrared and Raman-active modes within the $\mathrm{CH}$ fundamentals. This observation has consequences for the decay (relaxation) process which starts-after the pump pulses left the medium - from a reservoir of two or more energy states.

In the second process the vibrational energy is transferred from the $\mathrm{CH}$ stretching modes $\left(-3000 \mathrm{~cm}^{-1}\right)$ via overtones and combination modes to lower energy states. An intramolecular anharmonic coupling (a Fermi resonance) between neighboring energy states is of major importance in this respect. The degree of a Fermi resonance in a certain frequency range manifests itself in the infrared and Raman spectra. Overtones and higher-order combination modes "borrow" intensity from the $\mathrm{CH}$ stretching modes. We shall use the intensity ratio $R$ between the final and initial states, taken from an infrared or Raman spectrum, as a measure of the Fermi resonance mixing. Recently, Fendt, Fischer, and Kaise ${ }^{1}$ derived a formula for estimating the lifetime $T_{1}$ of a vibrational state:

$$
T_{1} \cdots N(1-R)^{2} R^{-1} \exp (01 /(I))^{*}:{ }^{*} T_{:}(j)
$$

where $N$ corresponds to the number of initially excited states and $T_{2}(f)$ is the dephasing time of the final state. This time can be estimated from the Raman line width $\Delta \nu$ as $T_{2}(f)=(2 \pi c \Delta \nu)^{-1}$, where $T_{2}(f)$ is equal to $T_{2} / 2$ measured in coherent Raman experiments. ${ }^{3}$

The frequency $\omega$ represents the energy difference between the initial and final states, whereas the frequency $\Omega$ has a value close to $100 \mathrm{~cm}^{-1}$. In some cases a complex band structure prevents determination of $T_{2}(f)$. Moreover, the above equation for $T_{1}$ breaks down for a very strong vibrational coupling when the Fermi resonance interaction becomes so strong that the energy transfer process is very fast, i.e., it occurs within the excitation pulse duration.

As an example, we shall report the data on two molecules: $\mathrm{CH}_{2}=\mathrm{CCl}_{2}$ and trans $-\mathrm{CHCl}=\mathrm{CHCl}$, the infrared absorption and Raman spectra of which are shown in Fig. 2 (Ref. 4). The two molecules have very similar structures: they differ only by a change in the positions of two atoms. Figure $3 \mathrm{a}$ shows the symmetric $\left(\nu_{1}\right)$ and asymmetric $\left(\nu_{7}\right)$ stretching vibrations of the $\mathrm{CH}_{2}$ group in $\mathrm{CH}_{2}=\mathrm{CCl}_{2}$ in a strong Fermi resonance with combination modes. In particular, there is a strong Fermi resonance between the modes $\nu_{1}$ and $\nu_{2}+\nu_{3}$, both of the $A_{1}$ symmetry, and also between $\nu_{7}$ and $\nu_{2}+\nu_{6}+\nu_{11}$, both of which are of the $B_{1}$ symmetry. ${ }^{4}$ This observation suggests that we have to consider two decay channels for the relaxation of the stretching modes of $\mathrm{CH}_{2}$. In the case of the $\nu_{1}-v_{2}+\nu_{3}$ process we can estimate the intensity ratio $R=0.2 \pm 0.05$ and calculate $T_{2}(f)=0.3$ psec employing the Raman line width $\Delta \nu=17 \mathrm{~cm}^{-1}$. For $N=2$ (two $\mathrm{CH}_{2}$ modes) and $\omega=40 \mathrm{~cm}^{-1}$, we find from Eq. (1) that $T_{1}=7.8 \pm 3$ psec.

In the case of the second decay channel $v_{7} \rightarrow \nu_{2}+\nu_{6}+$ $\nu_{11}$, the parameters are $R=0.6 \pm 0.1, T_{2}(f)=0.3 \mathrm{psec}$, $N=2$, and $\omega=45 \mathrm{~cm}^{-1}$, which gives $T_{1}=4.5 \pm 1 \mathrm{psec}$. Adding the two decay rates we obtain an estimate of the

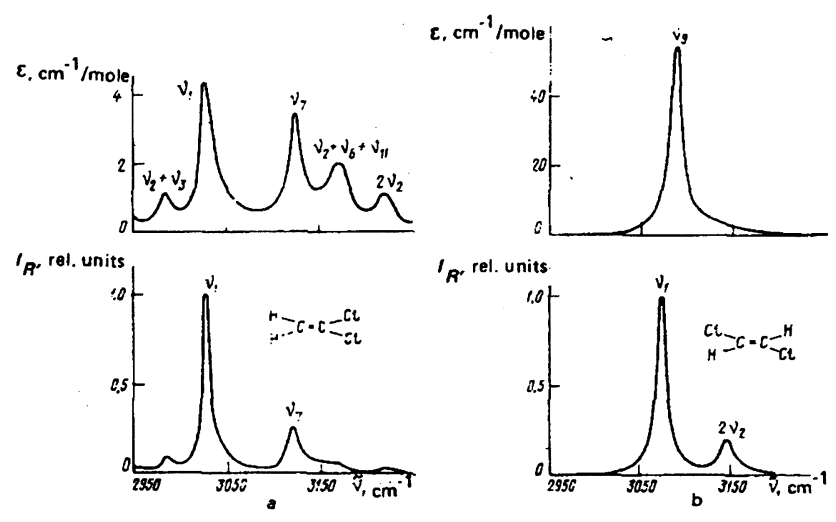

FIG. 2. Infrared and Raman spectra of $\mathrm{CH}_{2}=\mathrm{CCl}_{2}$ (a) and $\mathrm{CHCl}=\mathrm{CHCl}(\mathrm{b})$ in the spectral interval $2950-3250 \mathrm{~cm}^{-1}$ (here, $\varepsilon$ represents infrared absorption). 


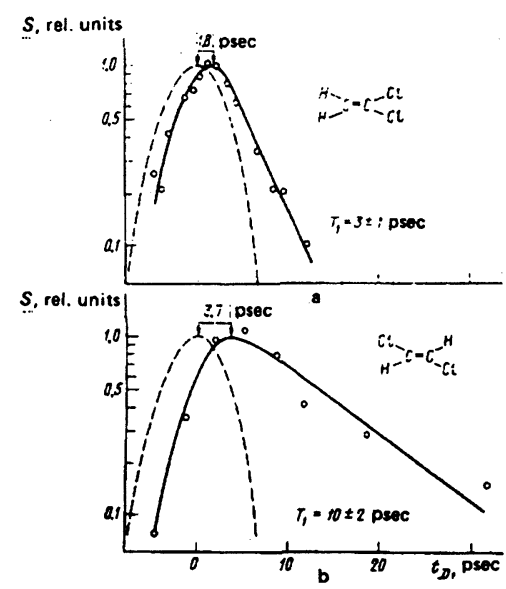

FIG. 3. Anti-Stokes scattering signal $S$ plotted as a function of the delay time of a probe pulse $t_{D}$ in solutions of $\mathrm{CH}_{2}=\mathrm{CCl}_{2}$ (a) and trans- $\mathrm{CHCl}=\mathrm{CHCl}$ (b) at a concentration 0.35 molar fractions: a) $\tilde{\nu}_{1}=3036 \mathrm{~cm}^{-1}, \tilde{\nu}_{2}=3036 \mathrm{~cm}^{-1}$; b) $\tilde{\nu}_{1}=3084 \mathrm{~cm}^{-1}$, $\tilde{\nu}_{2}=3073 \mathrm{~cm}^{-1}$. The dashed curves represent cross-correlation functions of the exciting (infrared) and interrogating (green) pulses.

relaxation time for the two $\mathrm{CH}_{2}$ stretching modes: $T_{1}$ $=2.9 \pm 0.6 \mathrm{psec}$.

Figure $3 a$ gives the results of the direct experimental determination of the time constant $T_{1}$ for the stretching modes of $\mathrm{CH}_{2}$. The $\nu_{1}$ mode at $3036 \mathrm{~cm}^{-1}$ is resonantly excited by an infrared pulse and the antiStokes scattering of the same $v_{1}$ mode is observed. The scattered Raman signal rises to a slightly delayed maximum during the excitation process and decays with a relaxation time $T_{1}=3 \pm 1$ psec. This experimental value is in excellent agreement with the calculated time constant $T_{1}$ discussed in the preceding paragraph. The dashed curves in Fig. 3 represent the cross-correlation. As pointed out above, they determine the zero point on the time axis and give a good indication of the time resolution of the apparatus.

Figure $2 b$ shows the infrared-active stretching mode $\nu_{9}$ and the Raman-active symmetric mode $v_{1}$ of trans$\mathrm{CHCl}=\mathrm{CHCl}$. Here we find a much weaker Fermi resonance. The Raman spectrum of Fig, $2 b$ suggests some anharmonic coupling between $\nu_{1}$ and $2 \nu_{2}$, both of the $A_{\text {s }}$ symmetry. Assuming that $R=0.15 \pm 0.02, T_{2}(f)$ $=0.3 \mathrm{psec}, N=2$, and $\omega=80 \mathrm{~cm}^{-1}$, we find that $T_{1}=13$ psec.

Figure $3 \mathrm{~b}$ gives the time dependence of the stretching modes of $\mathrm{CH}$ in trans $-\mathrm{CHCl}=\mathrm{CHCl}$. This molecule is excited via the $\nu_{9}$ mode at $3084 \mathrm{~cm}^{-1}$ and the population of the $\nu_{1}$ mode at $3073 \mathrm{~cm}^{-1}$ is monitored by anti-Stokes Raman scattering. The rapid rise of the Raman signal, i. e., the fast filling of the $\nu_{1}$ mode, provides clear evidence of fast energy exchange between the two $\mathrm{CH}$ fundamentals $\nu_{1}$ and $\nu_{\theta}$. The slow decay of the signal shows that the lifetime of two stretching modes of $\mathrm{CH}$ is long: $T_{1}=10 \pm 2 \mathrm{psec}$. This result is in good agreement with the value estimated above. A weak intramolecular coupling is responsible for the longer vibrational lifetime.

\section{DEPHASING TIME $T_{2}$}

In our investigations a specific vibrational mode was first coherently excited via the stimulated Raman effect and the time dependence of the vibrational excitation was monitored subsequently by a delayed and properly phase-matched probe pulse. The situation was straightforward for homogeneously broadened Raman transitions, ${ }^{5}$ when the probe signal decayed exponentially with time, providing directly the value of the dephasing time $T_{2}$. In a number of cases it was possible to show that the dephasing time accounted fully for the band width of the Raman transition $\Delta \tilde{\nu}_{R}$ in accordance with the relationship $T_{2}=1 / \pi c \Delta \tilde{\nu}_{R}$.

More recently, various theoretical papers have appeared to relate the collision-induced dephasing time to molecular parameters. ${ }^{7}$ In some cases the agreement between the theory and experiment is satisfactory.

\section{NEW RAMAN SPECTROSCOPY UTILIZING SHORT COHERENT EXCITATION AND SUBSEQUENT PROLONGED INTERROGATION}

We shall describe here a new spectroscopic technique $^{8,9}$ which provides information on homogeneously broadened transitions. New Raman lines can be detected in congested spectral regions and peak positions of Raman lines in liquids can be measured with unprecedented accuracy.

The idea underlying this new Raman technique is as follows. During a short transient excitation process the molecules in a liquid are driven by two light pulses separated by a frequency difference $\nu_{D}$. Raman transitions at and close to $\nu_{D}$ become excited and they have coherent amplitudes $Q_{f}$. This coherent excitation persists even when the two pump pulses have left the liquid sample. The molecules regain their resonance frequencies $\nu_{j}$ and the amplitude $Q_{j}$ decay with the characteristic dephasing time $T_{2 J}$. During the free relaxation process a third delayed long-duration probe pulse interacts with the coherently vibrating molecules. It generates coherently scattered Raman-shifted light carrying valuable information on the molecules. Only the molecules vibrating in phase during the probing process contribute to the coherent signal. The molecules which have suffered dephasing collisions vibrate out of step and are not observed. In the case of a suitable shape (for example, Gaussian) of the long-duration interrogating pulse the measuring time may be increased and the spectral resolution improved. This technique is based on short excitation and prolonged interrogation (SEPI) of molecular states. ${ }^{5,8,9}$

\section{EXPERIMENTAL SYSTEM FOR SHORT EXCITATION AND PROLONGED INTERROGATION SPECTROSCOPY}

The short dephasing times of vibrational modes in molecular liquids require picosecond pulses in order to measure SEPI spectra. It is convenient to use exciting pulses with a band width $\sim 10 \mathrm{~cm}^{-1}$ tuned in steps over a wider frequency range. For each excitation band the coherent spectrum should be recorded by a spectrometer or spectrograph with a sufficient resolution. Fig- 


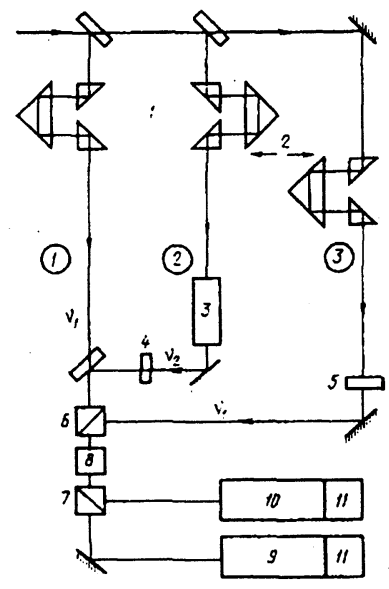

FIG. 4. Schematic diagram of a system used to study SE PI spectra of liquids: 1), 2) fixed and controlled optical delay lines; 3) Raman generator; 4) optical fllter; 5) $\lambda / 2$ plate; 6), 7) crossed polarizers; 8) investigated sample; 9), 10) spectrographs; 11) optical spectrum analyzer.

ure 4 shows schematically our apparatus. A single frequency-doubled pulse from a mode-locked Nd glass laser (shown in the top left-hand corner of Fig. 4) was used ${ }^{10}$; the duration of this pulse was $8 \mathrm{psec}$ and its frequency was $18990 \mathrm{~cm}^{-1}$. Two consecutive beam splitters were used to divide the pulse into three parts. The pulse in the first beam passed through a polarizer 6 and a sample, but was then blocked by a crossed polarizer 7 . The second beam altered the frequency to $\nu_{2}$ in a generator utilizing stimulated Raman scattering. A change in the working medium in the Raman generator readily produced pulses of different frequencies $\nu_{2}$. These pulses were blocked by the polarizer 7 and were spectrally monitored by a spectrograph 10 . In view of the transient nature of the generation process, the pulses of frequency $\nu_{2}$ were shorter by a factor of about three than the incident pulses at the frequency $\nu_{1}$ (Ref. 11). The two pulses in the first and second beams reached the sample simultaneously and excited coherently molecular vibrations because of transient stimulated Raman scattering at the difference frequency $\nu_{D}=\nu_{1}-\nu_{2}$. In the third optical path a delayed pulse with the polarization perpendicular to that of the pulses in the first and second beams was generated. This third pulse interacted with the coherently excited molecules in the sample producing a Raman-shifted signal pulse. Stokes scattering ensured that the three pulses traveled collinearly through the sample. In the case of anti-Stokes scattering, the probe pulse crossed the beam of the excited pulses at the phase-matching angle. The spectrum of the coherently scattered light was scattered using a two-meter spectrograph 9 and a cooled optical spectrum analyzer 11 . The system had a resolution of $0.2 \mathrm{~cm}^{-1}$ per channel and an absolute accuracy of the frequency scale of $0.4 \mathrm{~cm}^{-1}$.

\section{EXPERIMENTAL RESULTS ON LIQUID CYCLOHEXANE}

We carried out SEPI measurements on a number of organic and inorganic liquids. We shall give here the

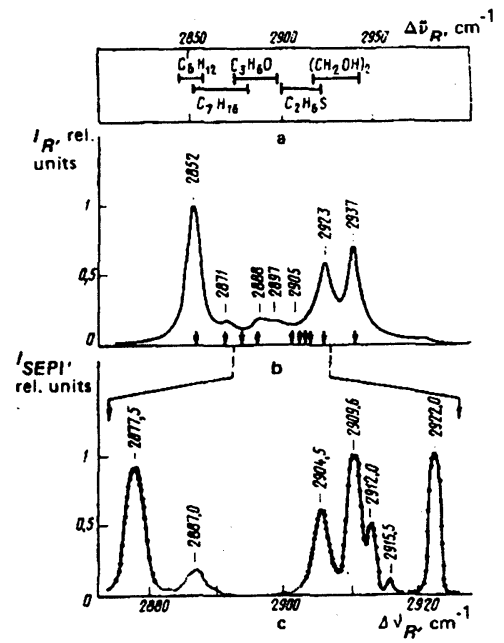

FIG. 5. Spectral intervals of various liquids used in the Raman generator (a), polarization spectra of the Raman scattering in $\mathrm{C}_{6} \mathrm{H}_{12}$ recorded with a resolution of $1 \mathrm{~cm}^{-1}$ (b) and three SEPI spectra obtained using three different liquids in the Raman generator (c). The arrows identify the positions of resonance lines observed in the SEPI spectrum.

results on liquid $\mathrm{CH}_{6} \mathrm{H}_{12}$ with broad and overlapping lines between 2850 and $2440 \mathrm{~cm}^{-1}$ in the usual spontaneous Raman spectrum. Figure $5 \mathrm{~b}$ shows the polarized (spontaneous Raman) spectrum. This spectrum was recorded using an argon laser and a Raman spectrometer with a resolution better than $1 \mathrm{~cm}^{-1}$. The three strong Raman bands observed in the spectrum were due to the $\mathrm{CH}$ stretching modes and the diffuse spectrum between 2860 and $2930 \mathrm{~cm}^{-1}$ was attributed to overlapping overtones and combination modes enhanced by a Fermi resonance with the fundamentals. ${ }^{12,13}$

Figure 5c shows three SEPI spectra on an expanded scale (by a factor of 3.7). Each spectrum was obtained using a single laser shot. On the right we plotted a sharp SEPI band corresponding to the $\mathrm{CH}$ stretching mode at $2923 \mathrm{~cm}^{-1}$. The small line width of $2.3 \mathrm{~cm}^{-1}$ enabled us to determine accurately the peak position as $2922.0 \pm 0.7 \mathrm{~cm}^{-1}$. The SEPI band was considerably narrower than the corresponding band in the spontaneous Raman spectrum of Fig. 5b. Moreover, the latter was asymmetric because of other weaker Raman transitions. The Raman transition at $2923 \mathrm{~cm}^{-1}$ was excited using ethylene glycol $\left(\mathrm{CH}_{2} \mathrm{OH}\right)_{2}$ in the Raman generator cell (Fig. 4).

The SEPI spectrum of Fig. 5c located to the left of the $2923 \mathrm{~cm}^{-1}$ line consisted of four Raman transitions. We resolved clearly the lines separated by just $2.5 \mathrm{~cm}^{-1}$. The four transitions were hidden under the wing of the strong Raman band at $2923 \mathrm{~cm}^{-1}$ and they could not be detected in the conventional Raman spectrum of Fig. 5b. The SEPI spectrum was obtained using an exciting pulse of frequency $\nu_{2}$ in the band $2900-2920 \mathrm{~cm}^{-1}$ (dimethyl sulfide $\mathrm{C}_{2} \mathrm{H}_{6} \mathrm{~S}$ was used in the Raman generator).

On the left hand side of Fig. 5c we plotted a SEPI spectrum obtained after excitation by a $\nu_{2}$ pulse with a 
spectral band width $2875-2890 \mathrm{~cm}^{-1}$ (propylene oxide $\mathrm{C}_{2} \mathrm{H}_{6} \mathrm{O}$ was used in the Raman generator). We found two distinct Raman bands at 2877.5 and $2887 \mathrm{~cm}^{-1}$, the former of which had not been detected earlier, because it was buried in the diffuse part of the conventional Raman spectrum (Fig. 5b).

The final identification of the new Raman lines observed in the interval $2870-2920 \mathrm{~cm}^{-1}$ has not yet been made. Inspection of the lower fundamental modes suggests overtones and combination modes in this frequency range. Of special interest is the new Raman line at $2912 \mathrm{~cm}^{-1}$, which coincides precisely with an infraredactive mode of the molecule. It appears that this is a forbidden Raman mode.

\section{ADDITIONAL OBSERVATIONS AND COMMENTS ON SHORT EXCITATION AND PROLONGED INTERROGATION SPECTRA}

The following points are important in the application of the SEPI technique.

The frequency positions of the observed Raman lines are independent of the excitation conditions, since we are observing freely relaxing molecules. We have tested this notion by exciting a sample with narrowor broad-band pulses of similar central frequency $\nu_{2}$. The advantage of a broad-band frequency spectrum of the incident pulse is the provision of initial conditions for several Raman transitions in congested spectral regions. One can then observe several Raman lines employing a single laser short (middle part of Fig. 5c).

The exciting and interrogating pulses in SEPI experiments should not overlap on the time scale in order to avoid the generation of a coherent signal via a nonresonant four-photon parameteric process. For this reason the delay time of the third probe pulse should be sufficiently large. One can roughly estimate the delay times of $t_{D}=20-25$ psec for dephasing times $T_{2 j}=1$ psec and Gaussian pulses of 8 psec duration. The SEPI spectra are observed with a good accuracy, approximately five orders of magnitude below the peak value at $t_{D}=0$.

The maxima of the SEPI spectra are not proportional to the Raman scattering cross section since the initial conditions of the exciting pulses and the dephasing times $T_{2,}$ are important in the observed magnitude of the generated signal. The SEPI spectra obtained after different delay times allow us to estimate the values of $T_{2 \mathrm{~s}}$.

The frequency precision of the generated Stokes spectrum depends on the frequency stability of the integrat- ing pulses. For the highest accuracy the frequency $\nu_{1}$ should be determined simultaneously with the SEPI spectrum: If an interrogating pulse has a chirped frequency spectrum, unwanted shifts appear in the SEPI spectra and such pulses should be avoided.

The scattering process may also occur on the antiStokes side of the spectrum. The disturbing interference found in stationary CARS spectroscopy does not occur in the delayed probing used in the SEPI spectroscopy. ${ }^{9,14}$

Finally, simultaneous determination of the coherent Stokes and anti-Stokes SEPI spectra allows us to eliminate the effects of a chirped probe pulse. In this way the absolute frequency position is determined with a high accuracy. ${ }^{9}$

\section{CONCLUDING REMARKS}

The data presented in this article demonstrate convincingly the usefulness of coherent and incoherent picosecond measurements. We can obtain molecular information and that cannot be found by other existing spectroscopic techniques.

${ }^{1}$ A. Fendt, S. F. Fischer, and W. Kaiser, Chem. Phys. 57, 55 (1981).

${ }^{2}$ A. Fendt, S. F. Fischer, and W. Kaiser, Chem. Phys, Lett. 82,350 (1981).

${ }^{3}$ A. Laubereau and W. Kaiser, Rev. Mod. Phys. 50, 607 (1978).

${ }^{4} \mathrm{C}$. Kolmeder, W. Zinth, and W. Kaiser, Chem. Phys. (in press).

${ }^{5}$ W. Zinth, H. J. Polland, A. Laubereau, and W. Kaiser, Appl. Phys. B 26, 77 (1981).

${ }^{6} A$. Sellmeier and W. Kaiser, Appl. Phys. 23, 113 (1980).

'S. F. Fischer and A. Laubereau, Chem. Phys. Lett. 35, 6 (1975); D. W. Oxtoby, Adv. Chem. Phys. 40, 1 (1979); R. M. Lynden-Bell, Mol. Phys. 33, 907 (1977).

${ }^{8}$ W. Zinth, M. C. Nuss, and W. Kaiser, Chem. Phys. Lett. (in press).

9w. Zinth, Opt. Commun. 34, 479 (1980).

${ }^{10}$ W. Zinth, A. Laubereau, and W. Kaiser, Opt. Commun. 22. 161 (1977).

${ }^{11}$ R. L. Carman, F. Shimizu, C. S. Wang, and N. Bloembergen, Phys. Rev. A 2, 60 (1970); S. A. Akhmanov, K. N. Drabovich, A. P. Sukhorukov, and A. S. Chirkin, Zh. Eksp. Teor. Fiz. 59, 485 (1970) [Sov. Phys. JETP 32, 266 (1971)].

${ }^{12}$ K. W. F. Kohlrausch and W. Wittek, Z. Phys. Chem. Abt. B 48, 177 (1941).

${ }^{13}$ K. B. Wiberg and A. Shrake, Spectrochim. Acta A 27, 1139 (1971).

${ }^{14}$ W. Zinth, A. Laubereau, and W. Kalser, Opt. Commun. 26, 457 (1978).

Edited by A. Tybulewicz 